\title{
Cosmetic Outcomes and Symmetry Comparison in Patients Undergoing Bilateral Therapeutic Mammoplasty for Breast Cancer
}

\author{
K. Gulis ${ }^{1,2}$ (1) - L. Rydén ${ }^{1,3} \cdot$ P. O. Bendahl ${ }^{3} \cdot$ T. Svensjö ${ }^{2}$
}

Accepted: 22 December 2020/Published online: 1 February 2021

(C) The Author(s) 2021

\begin{abstract}
Background Breast-reduction techniques are increasingly used in oncoplastic breast surgery. Bilateral therapeutic mammoplasty has the benefit of decreasing breast volume, enabling resection of larger tumors, and the potential to assure good postoperative symmetry. The aims of this study were to objectively asses the cosmetic outcomes of therapeutic mammoplasty in patients with breast cancer, using the breast cancer conservative treatment cosmetic results (BCCT.core) software, to compare this score with the surgeon's score and the patient's assessment, and to evaluate if other defined parameters have an impact on cosmetic outcomes. The secondary aim was to compare breast symmetry pre- and postoperatively.

Materials and Methods We enrolled 146 consecutive patients with primary breast cancer who underwent therapeutic mammoplasty between 2011 and 2018 in Kristianstad Central Hospital, Sweden. We retrospectively collected data from patients' records. We analyzed the BCCT.core score using postoperative photographs to objectively evaluate cosmetic outcomes on a four-grade scale and compared with preoperative photographs to evaluate symmetry. Cosmetic outcomes were also assessed subjectively by patients and surgeons, using a 10-point Likert scale.

Results The majority of patients (89\%) had good or excellent BCCT.core scores, which correlated with surgeons' scores, $\mathrm{rs}=-0.22(p<0.001)$. Overall, patients were more satisfied with the cosmetic outcomes than the surgeons $(p<0.001)$. Evidence supporting an association between the defined clinicopathological variables, for example, tumor size, and cosmetic outcomes, was weak.

Conclusion Therapeutic mammoplasty yields a very good cosmetic outcome, evaluated both by subjective and objective measurements. Importantly, symmetry can be improved in patients with asymmetry.
\end{abstract}

This study was presented as a poster/abstract with early results at the 2020 EBCC conference and the abstract text has been published in European Journal of Cancer.

Supplementary information The online version contains supplementary material available at (https://doi.org/10.1007/s00268020-05941-0).

K. Gulis

Kim.Gulis@med.lu.se

1 Department of Clinical Sciences Lund, Division of Surgery, Lund University, Lund, Sweden

\section{Introduction}

Breast cancer remains the most common cancer in women worldwide, and cosmetic results following surgery have gained increasing interest in recent decades, both with

2 Department of Surgery, Kristianstad Central Hospital, 29133 Kristianstad, Sweden

3 Department of Clinical Sciences Lund, Division of Oncology, Lund University, Lund, Sweden 
greater awareness among women and with the introduction of new surgical techniques.

Oncoplastic surgery was introduced more than 20 years ago, with the aim of optimizing both oncological safety and cosmetic outcomes, and studies provide evidence that oncological safety regarding local recurrence and survival are similar to rates after traditional breast-conserving surgery [1-4]. The principles of therapeutic mammoplasty were thoroughly described by McCulley and Macmillan in 2005 [5, 6]; however, subsequent studies involved small cohorts and mainly unilateral cases, very few with bilateral surgery only.

Currently, with 5-year survival rates for breast cancer often $>90 \%$ in the developed world, women have greater longevity after they are diagnosed, and having access to an early oncoplastic assessment affects the final cosmetic results. The ideal objective is that every woman diagnosed with breast cancer has the opportunity to obtain the best possible outcome and that each patient's satisfaction with the results is prioritized. However, the health and economic aspects of oncoplastic surgery must not be disregarded. The need for corrective surgery or re-excision after standard local excision can lead to two or more surgeries instead of one, with recovery times required after each surgery, which adds to the strain on already limited hospital resources. Consequently, there are several benefits regarding oncoplastic surgery for both society and patients.

Therapeutic bilateral mammoplasty is one technique used in oncoplastic surgery, and the technique is a valuable option to consider in patients with breast cancer who also have mammary hyperplasia and good health status [7, 8]. The alternative for these women is mastectomy in patients with larger tumors or multifocal tumors, sometimes with immediate or late reconstruction.

Recent studies have indicated that patient satisfaction with reconstructed breasts is lower compared with satisfaction after partial mastectomies, either following traditional surgery or with an oncoplastic approach [9]. Therefore, the ideal is to maintain the patient's own tissue, whenever possible. Women with mammary hyperplasia also have a higher risk of toxicity after radiation because of the higher required doses [10].

The objective of this study was to investigate cosmetic outcomes and breast symmetry in patients undergoing bilateral therapeutic mammoplasty for primary breast cancer using the breast cancer conservative treatment cosmetic results BCCT.core software for objective scoring, and comparing the BCCT.core score with the subjective opinion of both surgeons and patients. Our hypothesis was that outcomes following bilateral therapeutic mammoplasty are favorable both objectively and subjectively. The second objective was to investigate changes in symmetry pre- and postoperatively, with the hypothesis that symmetry would improve in patients with preoperative asymmetry. Several predefined variables (body mass index (BMI), multifocality, estimated percentage of breast volume excised, breast volume, extent, ptosis, mammaryjugular distance, axillary clearance, quadrant of the tumor location, smoking, and complications graded according to the Clavien-Dindo classification system [11] were measured to adjust for other relevant factors possibly affecting cosmetic outcomes.

\section{Material and methods}

All patients who underwent bilateral therapeutic mammoplasty with a diagnosis of primary breast cancer between January 2011 and August 2018 at Kristianstad Central Hospital were included in this retrospective observational cohort study.

The surgical procedure was offered to patients with breast cancer who also had marked asymmetry (canceraffected breast smaller than the other breast), hypertrophy, large tumor extent, or where the location of the cancer would result in unfavorable results with standard wide local excision. The planned approach and related factors for each patient were discussed in both pre- and postoperative multidisciplinary meetings.

Exclusion criteria were patients undergoing a secondary mastectomy, missing postoperative photographs, patients whose nipples were removed during surgery, and patients who died before the 1-year follow-up (Fig. 1).

Two patients in the cohort had missing preoperative photographs, and two had missing results from the 10-point Likert scale [12] evaluating the cosmetic outcomes; therefore, results for these patients could not be compared with the results of the BCCT.core scoring.

Clinicopathological data included in the prespecified case report form were collected from our hospital's patient record system (Melior ${ }^{\mathrm{TM}}$; Siemens Healthcare, Upplands Väsby, Sweden) and the operational record system Orbit5 ${ }^{\mathrm{TM}}$ (Every Healthcare Systems AB, Borås, Sweden). Data extraction was performed by a senior consultant in surgery (TS), and data for at least every tenth patient were independently validated by another surgeon (KG). All information was de-identified, and the information was kept in a coded database.

The clinicopathological data extracted from patients' records were pre- and postoperative photographs and patients' age, BMI, breast size, and previous diseases. We also collected data describing the tumor characteristics and patients' perioperative data, namely mammographic and ultrasonographic size, location of the tumor in the breast, and duration of surgery. The complete list of extracted variables is provided in Supplement 1. Age, tumor size, and 


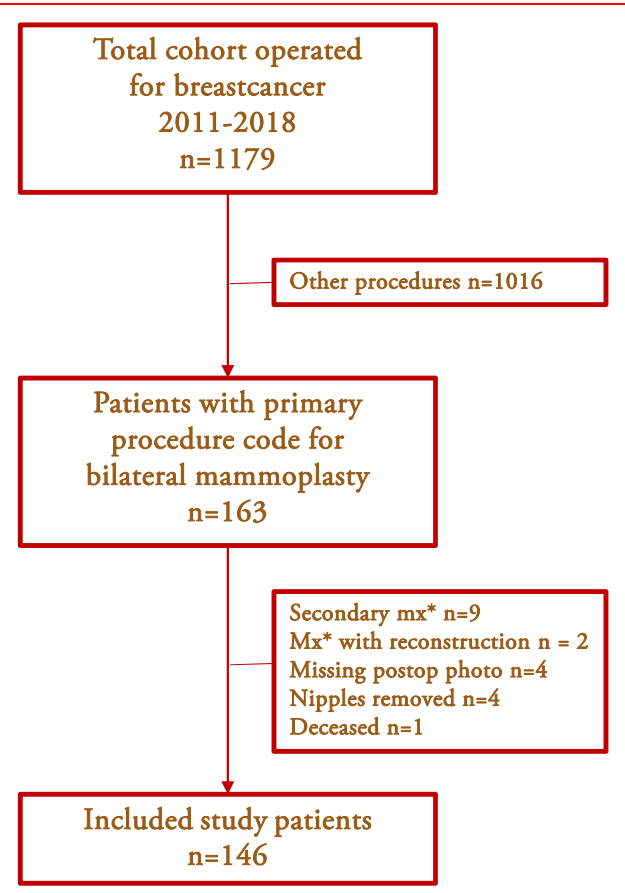

Fig. 1 Flowchart of the study $* \mathrm{Mx}=$ mastectomy

positive lymph node status were compared between the included patients and the complete cohort of patients undergoing breast-conserving surgery for breast cancer in our hospital from 2011 to 2018. These data were extracted from the National Swedish Breast Cancer Registry [13].

Before and approximately 1 year after surgery, the surgeon photo-documented patients' breasts in a frontal projection using an IXUS 95 or 100 camera (Canon Inc., Tokyo Japan). Breast volumes were measured by the surgeon using volume cups at the time of diagnosis [14].

We used postoperative photographs to evaluate cosmetic outcomes and analyzed the preoperative and postoperative photographs to compare pre- and postoperative symmetry; both evaluations were performed using the BCCT.core software $[15,16]$. This software evaluates postoperative cosmesis according to specific indices such as asymmetry, color, and scar visibility, to provide an overall cosmetic score, with outcomes graded as 1 (excellent), 2 (good), 3 (fair), or 4 (poor). Symmetry was evaluated quantitatively according to the percentage breast retraction assessment, which is a value calculating the symmetry of the distance between the jugular notch and mamilla in each breast, extracted from the BCCT.core score [17].

Cosmetic outcomes were also evaluated at the 1-year follow-up by all included patients and by the treating physicians. The evaluation was performed using a 10-point Likert scale for cosmetic outcomes, and the specific question asked by the treating physician was "How would you rate the cosmetic outcome on a scale to 1 to 10 , where
1 is the worst possible outcome and 10 is the best possible outcome." The results were registered for patients' and surgeons' evaluations, separately.

All patients were recommended to undergo postoperative radiotherapy according to Sweden's national guidelines. Complications were recorded at the first follow-up visit, approximately 14 days postsurgery, and at the 1-year follow-up. All complications were evaluated by a surgeon.

Complications were recorded for each patient according to the Clavien-Dindo classification system and the presence of complications on the breast cancer-affected side and the contralateral side, separately.

The correlation between the dichotomized BCCT.core scores and patients' and surgeons' scores was compared using Spearman's rank correlation coefficient $\left(\mathrm{r}_{\mathrm{s}}\right)$. Significance was analyzed using the Mann-Whitney $U$ test. Wilcoxon's matched-pairs signed-rank sum test was used to compare surgeons' and patients' scores, and also to analyze whether the breast affected with cancer had the same cosmetic outcomes as the contralateral breast.

McNemar's test was used to analyze whether the breast affected with cancer had the same frequency of postoperative complications as the contralateral breast, and we performed ordinal regression to evaluate the effects of the prespecified variables on the ordinal outcome BCCT.core score. The BCCT.core score was also dichotomized to excellent/good vs. fair/poor, and then analyzed with logistic regression.

IBM SPSS Statistics for Windows (IBM Corp., Armonk, NY) was used for the statistical analyses. $P$ values should be interpreted as level of evidence against each null hypothesis tested rather than as significant or not according to a cutoff. We made no adjustments for multiple testing. The study was registered in ISRCTN, identification number 82786416, and ethical approval was obtained from the Regional Ethics Review Board at Lund University, Sweden (2018/827). The study had an opt-out option for the patients implemented by an advertisement in the local newspaper in Kristianstad county.

\section{Results}

The final study cohort constituted 146 patients, and the patients' and tumor demographics are shown in Table 1.

The median age was 64 years (range $34-90$ years); 39\% of the patients were obese (BMI $\geq 30$ ), and $12 \%$ were current smokers. The median breast volume was $1000 \mathrm{ml}$, and the most common indication for surgery was breast hypertrophy. The most prevalent location of the tumor was the superior lateral quadrant. Operative data and additional demographic data appear in Supplement 2. 
Table 1 Patient and tumor demographics

\begin{tabular}{|c|c|c|c|c|c|}
\hline Patient demographics $(n=146)$ & No & $\%$ & Postoperative demographics $(\mathrm{n}=151)^{*}$ & No & $\%$ \\
\hline Age, years & & & EPBVE $^{\mathrm{d}}$ & & \\
\hline Median & 64 & & Median & 20 & \\
\hline Range & $34-90$ & & Range & $4.6-67.8$ & \\
\hline$<50$ & 22 & 15 & $\leq 20$ & 73 & 53 \\
\hline $50-59$ & 31 & 21 & $21-40$ & 45 & 33 \\
\hline $60-69$ & 62 & 42 & $41-60$ & 16 & 12 \\
\hline$\geq 70$ & 31 & 21 & $>60$ & 3 & 2 \\
\hline $\mathrm{BMI}^{\mathrm{a}}$ & & & Missing & 9 & \\
\hline Median & 28.5 & & Histological type & & \\
\hline Range & $19.9-48.2$ & & IDC & 106 & 70 \\
\hline$<22$ & 9 & 6 & ILC & 24 & 16 \\
\hline $22-24.9$ & 21 & 14 & Other types of IC & 10 & 7 \\
\hline $25-29.9$ & 59 & 40 & DCIS & 9 & 6 \\
\hline$\geq 30$ & 57 & 39 & LCIS and other types of in situ & 2 & 1 \\
\hline Smoking & & & Histological grade & & \\
\hline Non-smoker & 113 & 81 & I & 26 & 19 \\
\hline Ex-smoker & 10 & 7 & II & 65 & 49 \\
\hline Current smoker & 17 & 12 & III & 43 & 32 \\
\hline Missing & 6 & & Non-invasive & 11 & \\
\hline Breast size, ml & & & Complete remission & 6 & \\
\hline Median & 1000 & & Oestrogen $^{\mathrm{e}}>10 \%$ & & \\
\hline Range & $350-2200$ & & Yes & 113 & 82 \\
\hline$<800$ & 46 & 32 & No & 25 & 18 \\
\hline$\geq 800$ & 99 & 68 & Unknown & 13 & \\
\hline Missing & 1 & & Progesterone $^{\mathrm{f}}>10 \%$ & & \\
\hline MJ distance, $\mathrm{cm}$ & & & Yes & 90 & 65 \\
\hline Median & 29.75 & & No & 48 & 35 \\
\hline Range & $22.5-40$ & & Unknown & 13 & \\
\hline Ptosis, cm & & & HER $2^{\mathrm{g}}$ & & \\
\hline Median & 5.5 & & Yes & 16 & 12 \\
\hline Range & $0-14$ & & No & 122 & 88 \\
\hline Indications $^{\mathrm{b}}$ & & & Unknown & 13 & \\
\hline Asymmetry & 40 & 27 & Ki $67>30 \%$ & & \\
\hline Hypertrophy & 79 & 54 & Yes & 41 & 27 \\
\hline Multifocality & 25 & 17 & No & 110 & 73 \\
\hline Tumor size & 47 & 32 & Unknown & 12 & \\
\hline Tumor location & 16 & 11 & Lymph node status & & \\
\hline Re-excision & 7 & 5 & Benign 7 & 112 & 74 \\
\hline Ptosis & 24 & 16 & Metastasis & 39 & 26 \\
\hline Quadrant $^{\mathrm{c}}$ & & & Tumor size (mm) & & \\
\hline SMQ & 42 & 28 & Median & 18 & \\
\hline SLQ & 71 & 47 & Range & $0.5-58$ & \\
\hline ILQ & 20 & 13 & Extent $(\mathrm{mm})^{\mathrm{h}}$ & & \\
\hline IMQ & 14 & 9 & Median & 23 & \\
\hline
\end{tabular}


Table 1 continued

\begin{tabular}{llllll}
\hline Patient demographics $(\mathrm{n}=146)$ & No & $\%$ & Postoperative demographics $(\mathrm{n}=151) *$ & No \\
\hline Central & 4 & 3 & Range & $3-149$ \\
\hline
\end{tabular}

${ }^{\mathrm{a}}$ Body mass index

${ }^{\mathrm{b}}$ Multiple indications can be applied for each patient

${ }^{\mathrm{c}}$ SMQ:superior medial quadrant, SLQ:superior lateral quadrant, ILQ:inferior lateral quadrant,IMQ:inferior medial quadrant

${ }^{\mathrm{d}}$ Estimated percentage breast volume excised

${ }^{\mathrm{e} O e s t r o g e n}$ receptor status

${ }^{\mathrm{f}}$ Progesterone receptor status

${ }^{\mathrm{g}} \mathrm{HER} 2$ receptor status

${ }^{\mathrm{h}}$ Total size of tumor area, including DCIS

*Higher number due to bilateral cancers

Table 2 Demographics for all patients with primary breast cancer, operated in Kristianstad between 2011 and 2018 with breast-conserving surgery, compared to the study cohort

\begin{tabular}{lll}
\hline Demographics & $\begin{array}{l}\text { Total cohort } \\
(\mathrm{n}=1179)\end{array}$ & $\begin{array}{l}\text { Study } \\
\text { cohort }(\mathrm{n}=146)\end{array}$ \\
\hline $\begin{array}{l}\text { Age (median + range) } \\
\begin{array}{c}\text { Tumor size } \\
\text { (median }+ \text { range) }\end{array}\end{array}$ & $64(27-90)$ & $64(34-90)$ \\
$\begin{array}{l}\text { Positive lymph nodes } \\
\text { mm }(0-140)\end{array}$ & $18 \mathrm{~mm}(0.5-58)$ \\
\hline
\end{tabular}

Compared with all patients who underwent breast-conserving surgery in our hospital during the same years as our cohort, age had good conformity. The larger tumor size in our cohort was expected considering the patient selection criteria (Table 2).

The overall rate of complications classified according to the Clavien-Dindo system was $27 \%$, and most complications were grade $1-3$. The breast affected with cancer showed a higher frequency of complications (20/146) compared with the contralateral breast (10/146) (Table 3). There was weak evidence for a relevant difference between sides, using McNemar's test $(p=0.10)$.

The BCCT.core scores showed that $89 \%$ of all assessed patients received a result of good or excellent. None got a result of poor (Fig. 2).

Both surgeons and patients recorded high scores for general cosmetic outcomes, with a median value for the surgeons of $8 / 10$ and $9 / 10$ for the patients; median scores were identical for the cancer-affected breast and the contralateral breast (Table 3).
Table 3 Complications according to Clavien-Dindo Classification, any complications recorded for each breast, types of complications and cosmetic outcome for BCCT.core and Likert scales

\begin{tabular}{|c|c|c|}
\hline & No & $\%$ \\
\hline \multicolumn{3}{|l|}{ Clavien-Dindo Classification } \\
\hline 0 & 107 & 73 \\
\hline 1 & 12 & 8.2 \\
\hline 2 & 1 & 0.7 \\
\hline 3 & 19 & 13 \\
\hline 4 & 6 & 4.1 \\
\hline 5 & 1 & 0.7 \\
\hline \multicolumn{3}{|l|}{ Postoperative breast complications* } \\
\hline No complication in any breast & 109 & 75 \\
\hline Cancer-affected breast & 20 & 14 \\
\hline \multicolumn{3}{|l|}{ only } \\
\hline Contralateral breast only & 10 & 6.8 \\
\hline Bilateral complication & 7 & 4.8 \\
\hline \multicolumn{3}{|l|}{ Types of complications } \\
\hline Wound defects & 13 & 8.6 \\
\hline Bleeding & 11 & 7.3 \\
\hline Wound infections & 6 & 4.0 \\
\hline Seroma & 5 & 3.3 \\
\hline \multicolumn{3}{|l|}{ BCCT.core } \\
\hline Excellent & 40 & 27 \\
\hline Good & 90 & 62 \\
\hline Fair & 16 & 11 \\
\hline Poor & 0 & \\
\hline Likert scale & Median (range) & \\
\hline Surgeon & $8(3-10)$ & \\
\hline Patient & $9(1-10)$ & \\
\hline
\end{tabular}

separately recorded for each breast 


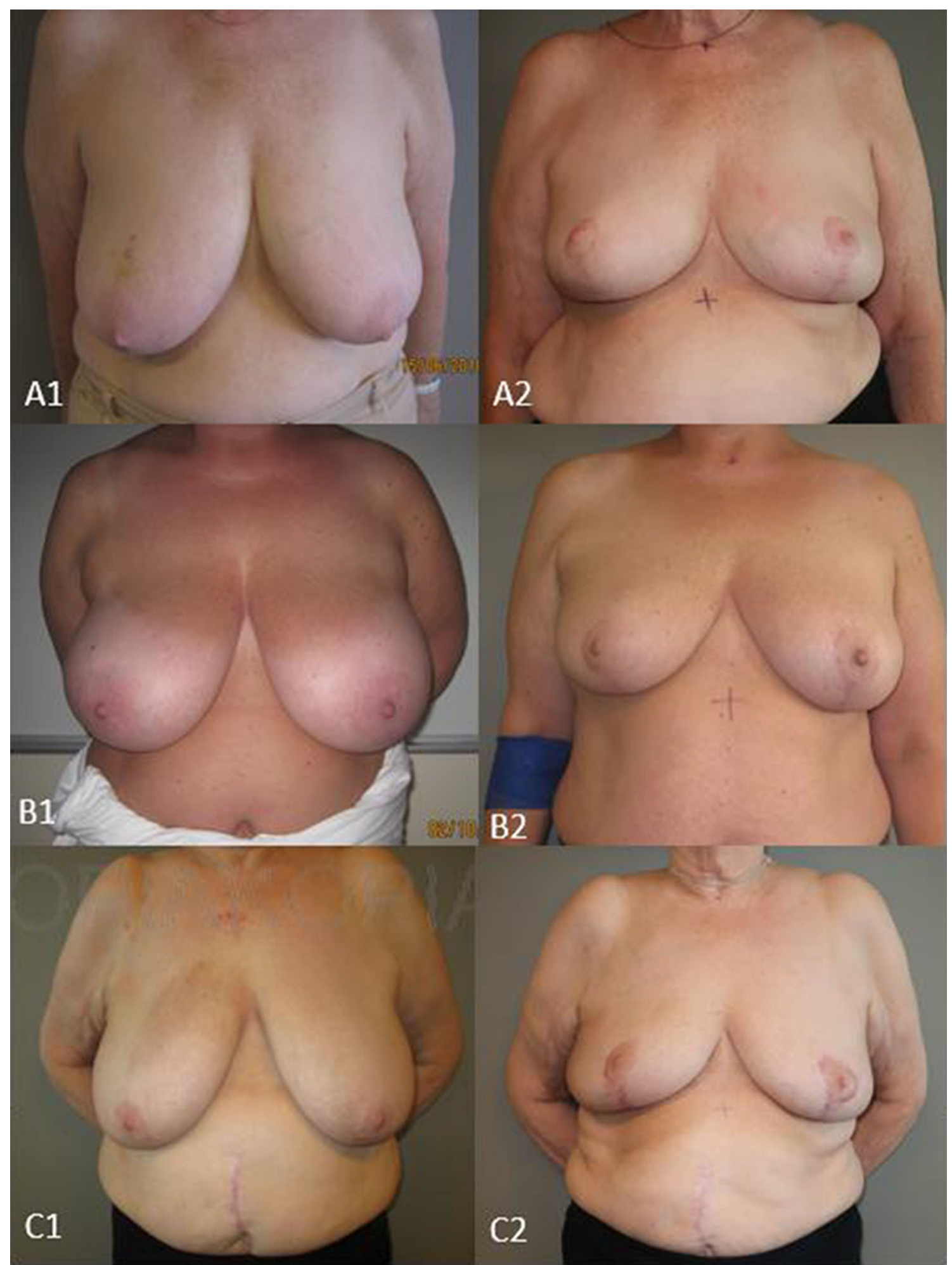

Fig. 2 Pre- and postoperative picture by different BCCT.core scores. A2: BCCT.core Excellent”,B2: "BCCT.core "Good" C2: BCCT.core "Fair" 
The correlation between the BCCT.core score and the surgeons' scores was $\mathrm{r}_{\mathrm{s}}=-0.23,(p<0.001)$. The negative value is because the scales in the BCCT.core system and the Likert scale are reversed.

Interestingly, patients were more satisfied with the outcomes than the surgeons $(p<0.001)$. When evaluating the cosmetic results for each breast separately, the Wilcoxon matched-pairs signed-rank test results indicated that patients were more satisfied with the cosmetic outcomes for the contralateral breast $(p=0.04)$. However, the surgeons' scores showed weak evidence supporting a relevant difference regarding the cancer-affected breast over the contralateral breast $(p=0.065)$.

Overall, symmetry showed no major change postoperatively in either direction, when we included the entire study cohort. However, in a subgroup analysis of five patient's with $\geq 25 \%$ asymmetry preoperatively, there was a positive change in the percentage breast retraction assessment value postoperatively, indicating improved symmetry (Supplement 3).

The prespecified variables showed weak evidence supporting an effect on cosmetic outcomes using the BCCT.core score, in our study, both with ordinal regression and when BCCT.core scores were dichotomized and analyzed with logistic regression (Table 4, Supplement 4).

Contralateral symmetry surgery was made immediately for several reasons. The main one was that the patient will not need a second surgery, and with two surgeons it does not prolong the surgery. Some patients also had mammary hyperplasia and immediate correction is favorable for these patients who will not have to wait for a symmetry correction with different sized breast. Since we have not seen any significant difference in symmetry between the breasts postoperatively, the cosmetic results are satisfactory even though $97 \%$ of the patients had radiotherapy to the affected breast.

\section{Discussion}

The strength of this study is its large cohort of patients undergoing pure bilateral surgery $(n=146)$ and the three predefined outcome measures for cosmetic outcome (BCCT.core, and patients' and surgeons' assessments). Previous studies evaluating patients undergoing bilateral breast cancer surgery [18-22] involved smaller cohort sizes of 20-82 patients, outcome measures were limited to one or two assessment methods, and only two studies used BCCT.core scores as an objective evaluation model for cosmetic outcomes $[20,21]$. In summary, our results confirmed previous findings of very favorable cosmetic outcomes after therapeutic mammoplasty in patients with primary breast cancer, with low surgical complication rates
Table 4 Predictors of cosmetic outcome in relation to BCCT.core univariate logistic regression

\begin{tabular}{|c|c|c|}
\hline Logistic regression & OR $(95 \% \mathrm{CI})$ & $p$ \\
\hline \multicolumn{3}{|l|}{ BMI, grouped } \\
\hline$<22$ & ref & \\
\hline $22-24.9$ & $0.37(0.043-3.141)$ & 0.36 \\
\hline $25-29.9$ & $0.40(0.067-2.359)$ & 0.30 \\
\hline$>30$ & $0.41(0.069-2.453)$ & 0.33 \\
\hline \multicolumn{3}{|l|}{ Smoking } \\
\hline None & ref & \\
\hline Previous & $0.53(0.064-4.326)$ & 0.55 \\
\hline Active & $2.10(0.400-11.078)$ & 0.38 \\
\hline Volume (ml) & $1.00(0.998-1.001)$ & 0.47 \\
\hline Ptosis $(\mathrm{cm})$ & $1.00(0.832-1.215)$ & 0.96 \\
\hline Mammary-jugular distance $(\mathrm{cm})$ & $0.90(0.771-1.052)$ & 0.18 \\
\hline \multicolumn{3}{|l|}{ Axillary clearance* } \\
\hline Yes & ref & \\
\hline No & $0.44(0.094-2.029)$ & 0.29 \\
\hline \multicolumn{3}{|l|}{ Quadrant } \\
\hline SMQ & ref & \\
\hline SLQ & $0.32(0.073-1.432)$ & 0.14 \\
\hline ILQ & $3.00(0.786-11.445)$ & 0.11 \\
\hline IMQ & $1.17(0.200-6.822)$ & 0.86 \\
\hline Central & - & - \\
\hline \multicolumn{3}{|l|}{ Multifocal* } \\
\hline Yes & ref & \\
\hline No & $0.52(0.112-2.426)$ & 0.34 \\
\hline Extent (mm) & $0,993(0.967-1.020)$ & 0.60 \\
\hline EPBVE (\%) & $0.99(0.953-1.030)$ & 0.64 \\
\hline \multicolumn{3}{|l|}{ Clavien-Dindo } \\
\hline No complication & ref & \\
\hline Mild (I-II) & $1.59(0.311-8.104)$ & 0.58 \\
\hline Severe $3<$ & $1.14(0.294-4.414)$ & 0.96 \\
\hline
\end{tabular}

[18-23]. Importantly, we also showed that cosmetic outcomes were not influenced by confounding factors such as tumor size or BMI. Previous studies showed that quality of life improved in healthy women with mammary hypertrophy undergoing breast reduction surgery [23], and a similar effect was shown previously for breast cancer patients [24]. The oncological aspects of breast reduction surgery have been addressed in a meta-analysis [1], and no significant difference was found regarding patients undergoing or not undergoing therapeutic mammoplasty. Additionally, a systematic review showed only positive effects of the oncoplastic approach [8], even after 20 years of follow-up [25].

A retrospective study by Dahlbäck et al. involving a Swedish cohort evaluated postoperative cosmetic results using BCCT.core scores in patients undergoing breast- 
conserving surgery. However, the study did not compare an oncoplastic surgery group because of the low frequency of this procedure (29/532 patients), in the study [26]. Our cohort had a higher median age compared with Dahlbäck et al.'s cohort (64 years vs 60 years, respectively) and larger tumor size (18 mm vs $15 \mathrm{~mm}$, respectively). Comparing the results in Dahlbäck et al.'s study with our cosmetic outcomes, $15.8 \%$ of Dahlbäck et al.'s patients were graded excellent and $57.4 \%$ as good, whereas $27.4 \%$ of our patients were graded excellent and $61.6 \%$ as good. These results indicate the added value of an oncoplastic approach regarding cosmetic outcomes, when evaluated using BCCT.core scores.

The finding that neither tumor size nor the percentage excised breast volume had a relevant impact on cosmetic outcomes is important. These findings indicate that therapeutic mammoplasty is a good option even with large tumors requiring a considerable portion of the breast to be removed.

However, patient selection is an important factor in all oncoplastic surgery, and the indications should therefore be clearly defined to optimize outcome.

The Clavien-Dindo complication rate of $27 \%$ in our study is similar to rates reported in other studies using this grading system, with $28.7 \%$ in one cohort and as high as $40.5 \%$ in a cohort undergoing bilateral mammoplasty $[7,11]$. In our study, we studied the complication rates for each side, with a higher incidence of complications in the breast with cancer, and this has not been widely studied before.

One limitation in our study is the retrospective design, even though the surgeons' and patients' assessments of the cosmetic results were collected at the same time, at the 1-year follow-up visit. Additionally, the study period spanned 7 years, but the same senior surgeon performing the oncoplastic surgery was part of the surgical team for the entire study period. Furthermore, our results are derived from data from a single hospital in Sweden and therefore must be interpreted with some caution. However, comparing our results with those for the entire cohort of patients undergoing breast-conserving surgery during the same time period, results showed good age and tumor size coherence, which strengthens our study.

Cosmetic results must be satisfying to the surgeon, but also to the patient; therefore, it is important to include patient-reported outcomes, including the patient's assessment of the cosmetic results, after surgery. Most studies to date are retrospective studies investigating cosmetic outcomes and quality of life, including our study. This means that there is little knowledge regarding patients' baseline status, which is important when comparing follow-up results.
There is a need for prospective studies comparing baseline and postoperative data to determine how cosmetic outcomes and patients' quality of life change. Patients with low quality of life at baseline cannot be expected to have a high quality of life postoperatively, but it is important to investigate whether there is a positive effect of oncoplastic surgery compared with standard breast-conserving surgery and mastectomy. We will address this issue in an ongoing prospective trial, NCT04227613.

Bilateral therapeutic mammoplasty yielded very good cosmetic outcomes in patients with primary breast cancer evaluated objectively using a software (BCCT.core) and, importantly, by the patient. Patients were also more satisfied with postoperative cosmetic outcomes than the surgeons. In this study, we found no clinically relevant factors influencing the cosmetic outcome.

Acknowledgement The authors would like to give a special thanks to all the study patients operated during the inclusion period, the personnel working at the breast cancer department in Kristianstad Central Hospital and the statisticians at the National Swedish Breast Cancer Registry. The authors also thank the Funding of Clinical Research within the National Health Service (ALF) and The Research and Development Committee at the Northeastern Hospitals in Skåne for funding this project and also Jane Charbonneau, DVM, from Edanz Group (www.edanzediting.com/ac) for editing a draft of this manuscript.

Funding Open Access funding provided by Lund University. This study was funded by the governmental Funding of Clinical Research within the National Health Service (grant number 2018-40304) and by The Research and Development Committee at the Northeastern Hospitals in Skåne, Sweden (grant number 85001-2040).

\section{Compliance with ethical standards}

Conflict of interest The authors declare that they have no conflict of interest.

Data availability The raw data will be available upon reasonable request from other researchers.

Open Access This article is licensed under a Creative Commons Attribution 4.0 International License, which permits use, sharing, adaptation, distribution and reproduction in any medium or format, as long as you give appropriate credit to the original author(s) and the source, provide a link to the Creative Commons licence, and indicate if changes were made. The images or other third party material in this article are included in the article's Creative Commons licence, unless indicated otherwise in a credit line to the material. If material is not included in the article's Creative Commons licence and your intended use is not permitted by statutory regulation or exceeds the permitted use, you will need to obtain permission directly from the copyright holder. To view a copy of this licence, visit http://creativecommons. org/licenses/by/4.0/. 


\section{References}

1. Kosasih S, Tayeh S, Mokbel K et al (2020) Is oncoplastic breast conserving surgery oncologically safe? A meta-analysis of 18,103 patients. Am J Surg 220(2):385-392

2. Papanikolaou IG, Dimitrakakis C, Zagouri F et al (2019) Paving the way for changing perceptions in breast surgery: a systematic literature review focused on oncological and aesthetic outcomes of oncoplastic surgery for breast cancer. Breast Cancer 26(4):416-427

3. Campbell EJ, Romics L (2017) Oncological safety and cosmetic outcomes in oncoplastic breast conservation surgery, a review of the best level of evidence literature. Breast Cancer (Dove Med Press) 9:521-530

4. De Lorenzi F, Hubner G, Rotmensz N, Bagnardi V et al (2016) Oncological results of oncoplastic breast-conserving surgery: Long term follow-up of a large series at a single institution: A matchedcohort analysis. Eur J Surg Oncol 42(1):71-77

5. McCulley SJ, Macmillan RD (2005) Planning and use of therapeutic mammoplasty-Nottingham approach. Br J Plast Surg 58(7):889-901

6. McCulley SJ, Macmillan RD (2005) Therapeutic mammaplastyanalysis of 50 consecutive cases. Br J Plast Surg 58(7):902-907

7. Di Micco R, O'Connell RL, Barry PA et al (2017) Bilateral mammoplasty for cancer: Surgical, oncological and patient-reported outcomes. Eur J Surg Oncol. 43(1):68-75

8. Piper ML, Esserman LJ, Sbitany H et al (2016) Outcomes Following Oncoplastic Reduction Mammoplasty: A Systematic Review. Ann Plast Surg 76(Suppl 3):S222-S226

9. Flanagan MR, Zabor EC, Romanoff A et al (2019) A Comparison of Patient-Reported Outcomes After Breast-Conserving Surgery and Mastectomy with Implant Breast Reconstruction. Ann Surg Oncol 26(10):3133-3140

10. Ratosa I, Jenko A, Oblak I (2018) Breast size impact on adjuvant radiotherapy adverse effects and dose parameters in treatment planning. Radiol Oncol 52(3):233-244

11. Panhofer P, Ferenc V, Schutz M et al (2014) Standardization of morbidity assessment in breast cancer surgery using the Clavien Dindo Classification. Int J Surg 12(4):334-339

12. Norman G (2010) Likert scales, levels of measurement and the "laws" of statistics. Adv Health Sci Educ Theory Pract 15(5):625-632

13. registry: AfSnbc. 2019 [Available from: https://www.cancercen trum.se/samverkan/cancerdiagnoser/brost/kvalitetsregister/

14. Hansson E, Manjer J, Ringberg A (2014) Reliability of plastic cups to measure breast volume. J Plast Surg Hand Surg 48(4):254-258

15. Cardoso JS, Cardoso MJ (2007) Towards an intelligent medical system for the aesthetic evaluation of breast cancer conservative treatment. Artif Intell Med. 40(2):115-126

16. Cardoso MJ, Cardoso J, Amaral N et al (2007) Turning subjective into objective: The BCCT core software for evaluation of cosmetic results in breast cancer conservative treatment. The Breast. 16(5):456-461

17. Fabry HFJ, Zonderhuis BM, Meijer S, Berkhof J et al (2005) Cosmetic outcome of breast conserving therapy after sentinel node biopsy versus axillary lymph node dissection. Breast Cancer Res Treat 92(2):157
18. Emiroglu M, Karaali C, Salimoglu S et al (2016) Oncoplastic reduction mammoplasty for breast cancer in women with macromastia: long term aesthetic, functional and satisfaction outcomes. Contemp Oncol (Pozn) 20(3):256-260

19. Munhoz AM, Montag E, Arruda EG et al (2006) Critical analysis of reduction mammaplasty techniques in combination with conservative breast surgery for early breast cancer treatment. Plast Reconstr Surg. 117(4):1091-1103 (discussion 104-7)

20. Santos G, Urban C, Edelweiss MI et al (2015) Long-Term comparison of aesthetical outcomes after oncoplastic surgery and lumpectomy in breast cancer patients. Ann Surg Oncol 22(8):2500-2508

21. Vella Baldacchino R, Bellizzi A, Madada-Nyakauru RN et al (2019) Assessment of breast symmetry in breast cancer patients undergoing therapeutic mammaplasty using the Breast Cancer Conservation Therapy cosmetic results software (BCCT core). Gland Surg. 8(3):218-225

22. Di Micco R, O'Connell RL, Barry PA et al (2017) Standard wide local excision or bilateral reduction mammoplasty in largebreasted women with small tumours: Surgical and patient-reported outcomes. Eur J Surg Oncol 43(4):636-641

23. Crittenden TA, Watson DI, Ratcliffe J et al (2019) Outcomes of Breast Reduction Surgery Using the BREAST-Q: A Prospective Study and Comparison with Normative Data. Plast Reconstr Surg 144(5):1034-1044

24. Losken A, Hart AM, Broecker JS et al (2017) Oncoplastic Breast Reduction Technique and Outcomes: An Evolution over 20 Years. Plast Reconstr Surg 139(4):824e-e833

25. Sanchez AM, Franceschini G, D'Archi S et al (2020) Results obtained with level II oncoplastic surgery spanning 20 years of breast cancer treatment: Do we really need further demonstration of reliability? Breast J 26(2):125-132

26. Dahlback C, Ullmark JH, Rehn M et al (2017) Aesthetic result after breast-conserving therapy is associated with quality of life several years after treatment Swedish women evaluated with BCCT core and BREAST-Q. Breast Cancer Res Treat 164(3):679-87

Publisher's Note Springer Nature remains neutral with regard to jurisdictional claims in published maps and institutional affiliations.

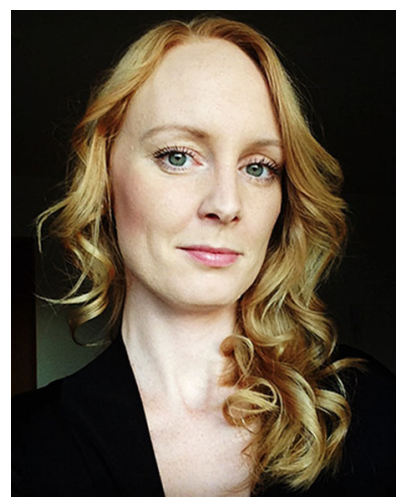

Kim Gulis was born and raised in Sweden. She obtained her medical degree from Copenhagen University and completed her residency in general surgery at Kristianstad Central Hospital. Her clinical interest is primarily in breast surgery, with a focus on oncoplastic surgery. Her research is focused on breastconserving surgery, cosmetic outcomes and the effects on quality of life. She is currently working on completing her thesis at the department of clinical sciences, Lund University. In her spare time, she enjoys travelling with her family and reading fiction novels. 